\title{
Oncological safety and cosmetic outcomes in oncoplastic breast conservation surgery, a review of the best level of evidence literature
}

This article was published in the following Dove Press journal:

Breast Cancer - Targets and Therapy

4 August 2017

Number of times this article has been viewed

\author{
Esther Jennifer Campbell' \\ Laszlo Romics ${ }^{2,3}$ \\ 'Department of Plastic Surgery, \\ Glasgow Royal Infirmary, ${ }^{2}$ Department \\ of Surgery, New Victoria Hospital \\ Glasgow, ${ }^{3}$ Department of Academic \\ Surgery, Glasgow Royal Infirmary, \\ Glasgow, Scotland, UK
}

\begin{abstract}
Oncoplastic breast conservation surgery (OBCS) is increasingly becoming part of routine breast cancer surgical management. OBCS may be viewed as an extension of standard breast conservation surgery for resecting tumors of larger sizes without compromising on cosmetic outcome, or as an alternative to mastectomy. High quality evidence to support the oncological safety and benefits of OBCS is lacking. This review will focus on the best available level of evidence and address key issues regarding oncological safety in OBCS, such as tumor resection margins and re-excision rates, local recurrence and patient outcome, postoperative complications and adjuvant therapy delivery, and briefly discuss cosmetic outcome in OBCS. Comparative observational studies and systematic review report no poorer outcomes compared with standard breast conservation surgery. More evidence needs to be generated to support the oncological safety and improved aesthetic outcome. Prospective data collection will significantly contribute to the generation of stronger evidence.
\end{abstract}

Keywords: oncoplastic breast conservation surgery, oncological safety, cosmetic outcomes, therapeutic mammoplasty, recurrence, survival

\section{Introduction - OBCS: rationale, definition, trends and evidence for practice}

Breast conservation treatment (BCT) defined as breast conservation surgery (BCS) with whole breast irradiation is the standard of care in the management of early breast cancer. The goal of BCT is tumor-free resection margins and good local control. An important secondary goal is a satisfactory cosmetic outcome as this is associated with both patient satisfaction and improved quality of life ${ }^{11}$ Poor cosmetic outcomes can affect up to $40 \%$ of patients undergoing BCT. ${ }^{25}$ There are many factors influencing the ultimate cosmetic outcome, including host factors, adjuvant therapy administered, tumor location in breast; however, the percentage of breast volume excised is the single most important factor influencing cosmetic outcome. ${ }^{11}$ How the breast looks after treatment is important because of the correlation between cosmetic outcome and the patients' anxiety and depression score, body image, sexuality and self-esteem. ${ }^{1}$

In the past decade, BCT has evolved to ensure both adequate oncological resection and good cosmetic outcome for patients with larger tumors. Increasing utilization of neoadjuvant therapy to enable tumor shrinkage and allow BCT is one strategy. Oncoplastic BCS (OBCS) with or without neoadjuvant therapy facilitates tumor excision with a wide margin of resection followed by immediate reconstruction of the defect (partial breast reconstruction), thus preserving a natural breast shape in
Correspondence: Laszlo Romics Department of Academic Surgery, Glasgow Royal Infirmary, Glasgow, G4 OSF Scotland, UK Email laszlo.romics@nhs.net 
woman and improving cosmetic outcome. Indications for OBCS include: anticipated poor cosmetic outcome with standard BCS (sBCS); large tumor in large breast; an alternative to mastectomy; or prevention of lymphedema, fibrosis and chronic pain that may be associated with irradiation in large-breasted woman. ${ }^{41}$ Additionally, as OBCS is increasingly being utilized as an alternative to mastectomy, with or without, immediate reconstruction, this approach may offer a lower complication rate compared with total mastectomy and reconstruction, particularly if radiotherapy is being given in the adjuvant setting. ${ }^{3,5,59}$ Potential benefits of this approach could be improved patient satisfaction, quality of life, as well as decreased health care costs compared with full breast reconstruction. ${ }^{67}$

OBCS is defined as level 1 and 2 techniques. ${ }^{9}$ Level 1 oncoplastic techniques does not require specialist plastic surgical techniques and is used to prevent deformities for tumors excisions that are $<20 \%$ of the breast volume and includes simple reshaping without skin excision and may require nipple recentralizing. Level 2 oncoplastic techniques should be considered when major volume loss is anticipated and are classified as volume displacement and volume replacement techniques. The majority of OBCS level 2 techniques utilize volume displacement techniques, which comprises tumor excision followed by reshaping of the breast parenchyma as well as reduction of the breast skin envelope. ${ }^{9}$ This is commonly referred to as therapeutic mammoplasty, and is often accompanied by a reduction of the contralateral breast to improve symmetry. Volume replacement OBCS techniques include latissimus dorsi (LD) myocutaneous flap, and various other pedicled flaps based on the use of intercostal artery or thoracodorsal artery perforators or a thoracoepigastric flap. Level 2 OBCS has traditionally been regarded as requiring specialized training in plastic surgical techniques.

OBCS has become widely accepted and adopted into routine clinical practice. Accurate national data on current utilization and practice of OBCS is limited. ${ }^{3}$ A recent study from the MD Anderson Cancer Center in the USA demonstrated that OBCS had a nearly fourfold increase in the percentage of all breast cancer surgeries performed (from 4\% to $15 \%$ ) between 2007 and 2014. In 2014, OBCS accounted for over $33 \%$ of all breast conservation surgeries. ${ }^{5}$ It is likely that similar increasing rates of OBCS procedures are being undertaken in Europe and the UK. The techniques of OBCS previously required either specialist oncoplastic training for breast surgeons or a combined approach by breast oncological surgeon and plastic surgeons. In the UK, at present, all general surgeons with a breast subspeciality interest require competency in mammoplasty techniques and involvement in pedicled flaps for certificate of completion of training (CCT). All newly appointed consultant breast surgeons in the UK will be competent in the techniques of mammoplasty.

Despite the widespread adoption of OBCS, there is limited high quality evidence to support the benefits of this approach. ${ }^{3,25,41,68}$ OBCS utilizes the principles of sBCS; however, the landmark prospective randomized trials that established the safety and efficacy of BCT mostly included patients with small tumors. ${ }^{19,63}$ Patients who were treated with OBCS often had larger tumors; in studies, over half the patients treated with OBCS had T2-T3 cancers. ${ }^{10,22,37-40,45,49,55,58,62}$ The evidence that cancers of these sizes can be safely treated with breast conservation is not robust in the classic prospective randomized trials. ${ }^{38}$ Only 599 patients with T2 cancers were randomized into the arm of breast conservation with radiotherapy in 3 trials published by van Dongen et al, Poggi et al and Fisher et al, although the latter had randomized up to $4 \mathrm{~cm}$ cancer size. ${ }^{20,47,60}$ Interestingly, patients with T1 cancers only were randomized by Veronesi et al and Arriagada et al. ${ }^{2,64}$ Hence, the classic randomized controlled trials do not provide sufficient evidence that breast conservation is safe in T2 cancers and above. Most published OBCS studies are small, single center, observational studies reporting (often inconsistent and heterogenous) outcomes. Comparative studies represent a higher level of evidence and currently this is the best available evidence. This literature review will examine the key issues in OBCS reported in observational comparative studies, including tumor resection margins and re-excision rates, local recurrence and patient outcome, postoperative complications and adjuvant therapy delivery, and briefly discuss cosmetic outcome in OBCS.

\section{Oncological safety: tumor resection margins and re-excision rates}

Involved resection margins are one of the most important factors associated with local recurrence after BCS and the standard surgical practice is to obtain clear margins even if this requires a second surgical procedure. The evidence base for surgical margins is continuously evolving and there is no universal consensus on what defines a positive margin. In the UK, most breast surgeons consider a $1 \mathrm{~mm}$ minimum clear radial margin for both ductal carcinoma in situ (DCIS) and invasive breast cancer. ${ }^{56}$ However, the Society of Surgical Oncology, the American Society for Radiation Oncology and the American Society of Clinical Oncology recently published a consensus statement on margins. ${ }^{43}$ This guidance differs from the Association of Breast Surgery guidance in 
that they recommend a $2 \mathrm{~mm}$ excision margin for DCIS. Involved surgical margins occur in $20 \%-40 \%$ of all standard $\mathrm{BCS}$ and 1 in $5 \mathrm{BCS}$ patients undergo a reoperation (including re-excision or completion mastectomy). ${ }^{25,29}$

OBCS allows wider oncological resections, and although wider negative margins are not associated with lower recurrence rates, advocates of OBCS argue that wider resections reduce positive margin rate and results in less re-excisions (or reoperations) compared with standard $\mathrm{BCS} .^{27,52} \mathrm{Re}$-excisions have the potential to delay adjuvant treatment, surgical complications and may compromise cosmetic outcome. Additionally, a further operation will cause stress for patients and their families, patient discomfort and increased health care costs. Thirteen comparative studies have compared OBCS with a control group, examining rates of positive margin involvement and reoperation rates (Table 1). 5,6,8,14,17,22,24,30,37,39,57 (PMID 26208579, 25121787). Not all studies report the positive margin rate and there is clear lack of consensus regarding the definition of positive margins. Additionally, not all studies report the re-excision or mastectomy conversion rate. Only 8 of these comparative studies report a statistically significant benefit in terms of negative margins and/or reoperation rate. The largest of these was a retrospective cohort study that included 1,177 patients treated with OBCS. ${ }^{5}$ The control arms included patients treated with $\operatorname{sBCS}(n=3,559)$, mastectomy only $(n=3,263)$ and mastectomy plus immediate reconstruction $(n=2,608)$. In terms of margin status, patients who underwent OBCS had significantly less positive or close margins $(5.8 \%)$ compared with $\mathrm{sBCS}(8.3 \%, P=0.04)$; the study did not report on re-excisions or reoperation rates. Chakravorty et al reported significantly less re-excision and mastectomy conversion in OBCS patients $(n=150)$ compared with $\operatorname{sBCS}(n=440) .{ }^{6}$ The remaining comparative studies reported significantly less tumor margin involvement and re-excision rates were limited by small patient numbers. Five comparative studies failed to demonstrate a benefit in terms of tumor-free margins and reoperations between OBCS and sBCS (Table 1).

A recent systematic review collectively evaluated over 5,000 patients treated with OBCS in 49 studies reported a weighted average positive margin rate of $10.8 \%$, re-excision rate of $6.0 \%$ and conversion to mastectomy rate of $6.2 \% .{ }^{13}$ This would suggest that the positive margin rate and subsequent re-operation rate is lower than $\mathrm{sBCS}$. Other systematic reviews have failed to conclude on benefit of tumor-free margins and lower re-excision rates in OBCS given the diverse and heterogenous study reporting with variation in the frequency of margin involvement ranging between $0 \%$ and $36 \%$ of patients. ${ }^{25,41,68}$ Additionally, in patients with positive margins, the subsequent management varied with re-excision rates of $11 \%-75 \%$, completion mastectomy rates of $8 \%-100 \%$, no further treatment or radiotherapy boost to tumor bed in some studies. ${ }^{41}$ It is clear from the current literature that the wider resections resulting from OBCS procedures does not obviate positive tumor margins and that the management of involved margins is not standardized. Oncoplastic volume displacement procedures are the most commonly employed OBCS procedure and this can result in displacement of the mammary tissue and hamper subsequent re-excision of the tumor bed necessitating conversion to mastectomy to ensure oncological safety and adequate tumor excision.

\section{Oncological safety - local recurrence and patient outcome}

The Oxford overview demonstrates that $75 \%$ of local recurrences occur within 5 years of surgery and confirmed the observation that for every 4 local recurrences prevented by adjuvant radiotherapy, 1 breast cancer death was prevented (4:1 ratio); this highlights the importance of local control in terms of patient survival. ${ }^{12}$ Rates of breast cancer local recurrence are falling. A $1 \%$ annual rate was considered acceptable; however, reported rates are now $<0.5 \%$ per annum.

Locoregional recurrence has historically been perceived as a failure of adequate local control; however, in the modern era, there is increasing recognition that locoregional recurrence is influenced by tumor biology. Meta-analysis has demonstrated that local recurrence after BCT for non-triple negative breast cancer have approximately halved the risk of local recurrence compared with triple negative breast cancer, and breast cancer subtype affects the number of locoregional events. ${ }^{4,35}$ Additionally, systemic therapy has a major impact on both local regional recurrence and distant recurrence. ${ }^{12,36}$

Patients treated with OBCS often have larger breast tumors. T2-T3 tumors comprise over half the OBCS patients treated in many studies. ${ }^{10,22,37-40,45,49,55,58,62}$ Large tumor size is a poor prognostic marker and may be a marker of time in situ or accelerated tumor growth and biological aggressiveness. It remains poorly defined whether OBCS represents an extension of the application of sBCS to larger tumors or whether a mastectomy was turned into conservation surgery with oncoplastic techniques. We recently compared the histopathological features of patients treated with OBCS, sBCS and mastectomy, with or without, immediate reconstruction $(\mathrm{Mx}$ \pm IR) in 1,000 consecutive operable breast cancer patients. ${ }^{37}$ Tumor size, grade, nodal status and hormone receptor expression were all significantly different from sBCS, being less favorable in all aspects after OBCS. The histological results 
Campbell and Romics

Dovepress

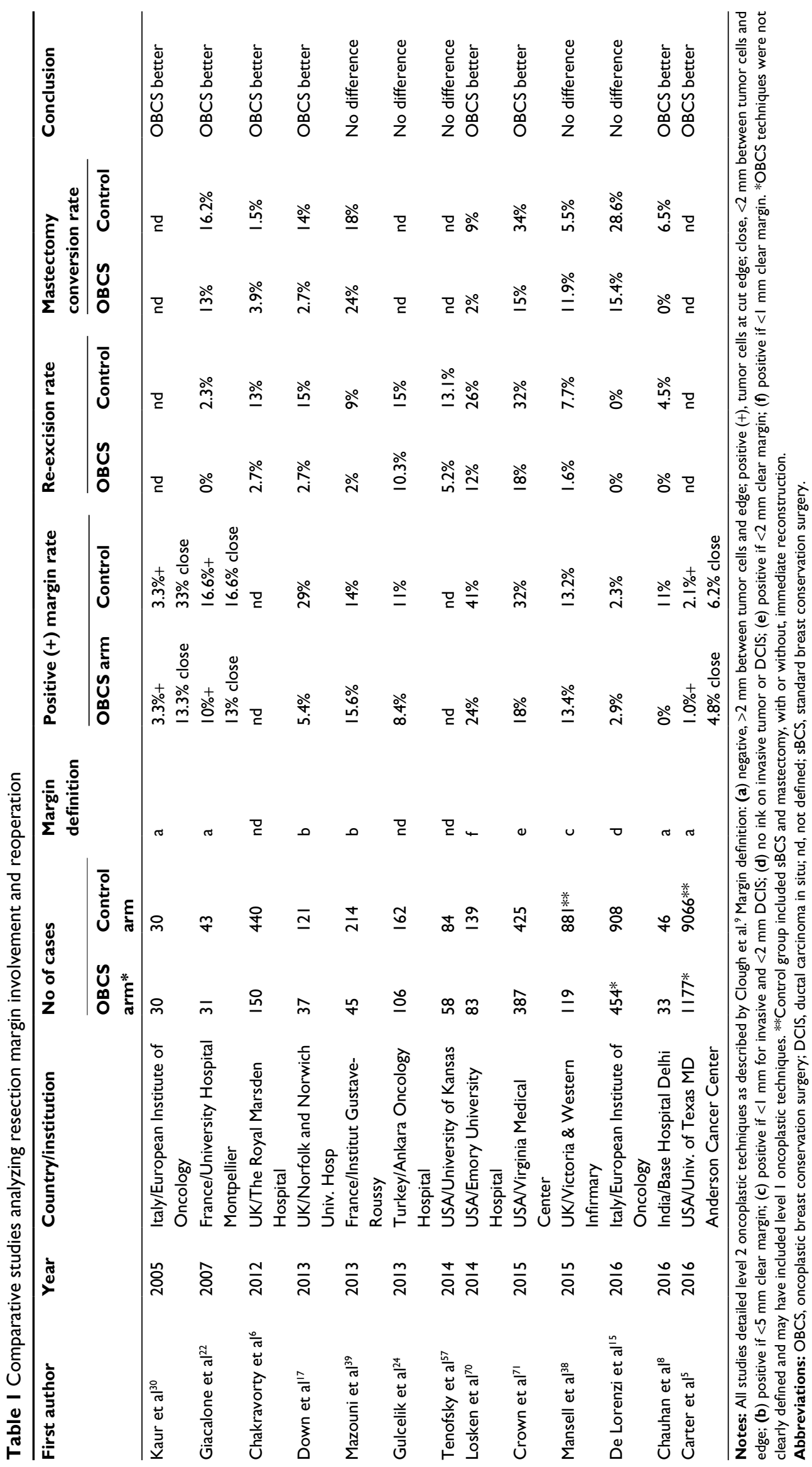

\begin{tabular}{llr}
\hline $\mathbf{5 2 4}$ & $\begin{array}{l}\text { submit your manuscript } \mid \text { www.dovepress.com } \\
\text { Dove press }\end{array}$ & Breast Cancer - Targets and Therapy 20I7:9
\end{tabular} 
(and adjuvant therapy application) were similar in patients treated with $\mathrm{Mx} \pm$ IR and OBCS. The striking similarities between OBCS and mastectomy patients' histopathology results suggests that, when analyzing recurrence rates and survival, this should be compared with both mastectomy and sBCS patients.

There is a lack of high level evidence supporting the oncological safety of OBCS in terms of local recurrence, patient disease-free survival (DFS) and breast cancer-specific survival. Prospective randomized trials are unlikely to ever be undertaken, given the complex ethical considerations. The current best available evidence is level 3, observational studies with control groups. To date, 8 comparative studies have been published that report on recurrence rates and survival, the ultimate measures of oncological safety (Table 2). ${ }^{5,6,8,14,15,24,38,39}$ Only 3 studies included local recurrence rates and survival compared with mastectomy patients and most studies are limited in terms of follow-up time.

Chakravorty et al reported equivalent safety in a retrospective comparative study that compared OBCS with sBCS. ${ }^{6}$ The OBCS group included significantly larger tumors, higher grade and more patients had received neoadjuvant chemotherapy. However, the OBCS also included a significant greater number of patients with noninvasive breast cancer. There was no difference in the adjuvant treatment therapy given and no significant difference in local relapse rates (OBCS $2.7 \%$ vs sBCS $2.2 \%$ ) or distant relapse (1.3\% OBCS vs $7.5 \%$ sBCS) at median follow-up of 28 months. Mazouni et al compared sBCS with OBCS after primary chemotherapy in a retrospective study with median follow-up of 46 months. ${ }^{39}$ They reported no significant difference in 5-year overall survival (OS; $96.2 \%$ OBCS vs $94.2 \%$ sBCS) or relapse-free survival ( $92.7 \%$ OBCS vs $92 \%$ sBCS). The groups were equivalent in terms of tumor size, grade, nodal disease; however, the OBCS had significantly less HER2 positive patients, more ER+, suggesting better breast cancer subtypes. Gulcelik et al performed a prospective study comparing quadrantectomy to therapeutic mammoplasty with a median follow-up of 33 months and reported no difference in DFS or OS. ${ }^{24}$ Between the groups, there was no significant difference in tumor size, ER status, HER2 status and adjuvant treatment given, although the study failed to detail tumor grade and nodal involvement in the 2 groups.

The largest comparative study is a retrospective singleinstitution study that included 9,861 consecutive patients diagnosed between 2007 and 2014 with a median follow-up of 3.4 years. ${ }^{5}$ Four groups were included: $\mathrm{sBCS}(\mathrm{n}=3,559)$, OBCS $(n=1,177)$, mastectomy only $(\mathrm{Mx})(\mathrm{n}=3,263)$ and

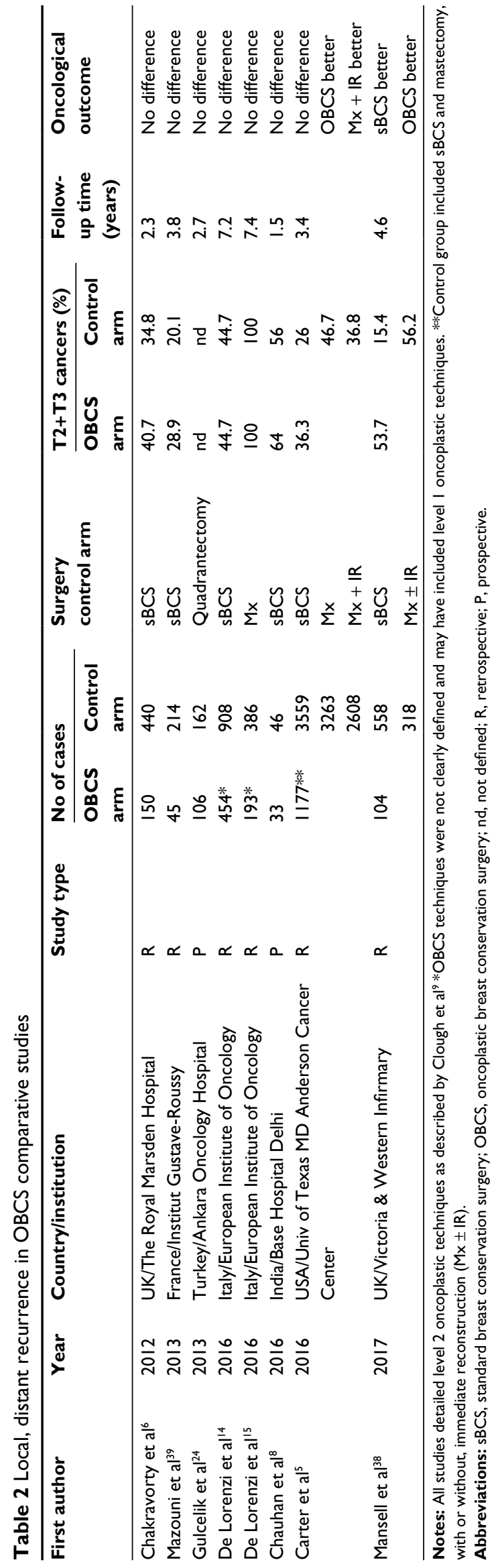


mastectomy plus immediate reconstruction $(\mathrm{Mx}+\mathrm{IR})$ $(n=2,608)$. Compared with sBCS $(n=3,559)$, patients undergoing OBCS $(n=1,177)$ had more aggressive diseases. There was no difference in the proportion of hormone receptor positive or triple negative patients in the OBCS group; however, they were significantly: younger in age, had larger tumors, more advanced disease stages, higher tumor grade, higher incidence of multifocality, node positivity, LVI, more HER2 positivity, more adjuvant chemotherapy administered and surprisingly less adjuvant hormonal therapy and adjuvant radiotherapy. Despite the marked differences in the clinicopathological features between sBCS and OBCS groups, there was no difference in 3-year OS (95.8\% OBCS vs $96.8 \%$ sBCS) and recurrence-free survival (94.6\% OBCS vs $96.1 \%$ sBCS). Comparing patients undergoing $\mathrm{Mx}+\operatorname{IR}(\mathrm{n}=2,608)$ with OBCS, noninvasive breast cancer and stage 0 was statistically more frequent in the $\mathrm{Mx}+\mathrm{IR}$ group, although there was no difference between $\mathrm{Mx}+\mathrm{IR}$ and OBCS in nodal stage or triple negative breast cancer. Ms + IR patients compared with OBCS did have higher grade tumors, higher incidence of multifocality, higher LVI, lower proportion of hormone receptor positivity, and higher number of HER2 positive tumors. More Mx + IR patients received adjuvant chemotherapy. Patients undergoing Mx + IR had significantly better 3-year OS (97.7\% Mx + IR vs 95.8\% OBCS, $P=0.0007)$ and recurrence-free survival $(96.6 \% \mathrm{Mx}+\mathrm{IR}$ vs $94.6 \% \mathrm{OBCS}$, $P=0.01)$. The authors accounted this difference in outcome to the larger proportion of patients with in situ or stage 0 disease in the Mx + IR group. The authors did not perform a direct statistical analysis comparing demographics of OBCS to Mx $(n=3,263)$, although Mx patients had the most advanced stage disease in the cohort, including $5 \%$ with metastatic disease. Unsurprisingly, the Mx had the worst patient outcome of all the groups. In multivariate analysis, when comparing surgical procedures, only Mx was significantly different from OBCS, with a hazard ratio over 2 times that of OBCS for death or recurrence. The authors concluded that OBCS does not disadvantage patients in terms of short-term outcomes when compared to sBCS or Mx. While this is the largest comparative study performed, the follow-up period in this study is too short to be truly meaningful in terms of local/distant recurrence or survival, especially given the heterogenous tumor pathology between the unmatched groups.

We analyzed 5-year local recurrence and distant recurrence rates in our cohort of consecutive treated operable breast cancer patients. Patients were treated with OBCS $(n=104), \operatorname{sBCS}(n=558)$ and Mx $\pm \operatorname{IR}(n=318) .{ }^{38}$ Within the OBCS group, patients were younger and tumor size, grade, nodal status and hormone receptor expression were all significantly different from sBCS, being less favorable in all aspects after OBCS. The histological results (and adjuvant therapy application) were similar in patients treated with $\mathrm{Mx}$ \pm IR and OBCS. There was no statistical difference in 5-year local recurrence rates between the 3 groups $(2 \%$ OBCS, $3.4 \% \mathrm{sBCS}, 2.6 \% \mathrm{Mx} \pm \mathrm{IR}$ ). In terms of distant recurrence, rates were significantly higher after $\mathrm{Mx} \pm \mathrm{IR}$ and OBCS (13.1\% Mx \pm IR, 7.5\% OBCS, 3.3\% sBCS, $P<0.001)$. The higher rates of distant recurrence after OBCS, compared with sBCS, most likely reflects the more advanced cancer pathology and biological aggressiveness in this group, and does not indicate that OBCS is unsafe oncologically; rather it needs to be compared with patients who undergo surgery for similar tumor pathology. Hence distant recurrence rates after mastectomy and OBCS were more alike.

A weakness of the above comparative studies is that the control groups were not matched and it was therefore difficult to make conclusions about oncological safety and patient outcome, given the heterogenous tumor pathology. De Lorenzi et al, published 2 case-matched studies comparing OBCS to sBCS and mastectomy for primary invasive breast cancer patients diagnosed in a single institution between 2000 and $2008 .{ }^{14,15}$ In the first study, OBCS ( $\left.=454\right)$ was compared with sBCS ( $n=908)$. Age at surgery, year of surgery and tumor size (including T1-T4) were the variables used for matching. In terms of clinicopathological features between the 2 groups, there was no significant differences in tumor histological type, grade, lymph node status, surgical margin involvement, tumor subtype (luminal A, B, ER+/HER+, HER2 enriched and triple negative), presence of perivascular invasion and adjuvant systemic therapy administered. The OBCS group had significantly more patients with multifocal disease. The median follow-up was 7.2 years. The OS was similar (91.4\% OBCS vs $91.3 \%$ sBCS at 10 years). The incidence of local recurrence was slightly higher on the OBCS group (3.2\% vs $1.8 \%$ at 5 years; $6.7 \%$ vs $4.4 \%$ at 10 years) but this was not statistically significant, and regional and distant events were similar between the groups. In the second study, OBCS $(n=193)$ was compared with mastectomy $(n=386)$ in patients with T2 $(2-5 \mathrm{~cm})$ invasive breast cancers. Over $90 \%$ of mastectomy patients had immediate reconstruction performed. Cases were matched using age at surgery, year of surgery and tumor subtype. In the mastectomy group, tumor multifocality was more frequent and tumors were significantly larger than in the oncoplastic group. For all other clinicopathological features not used in the matching algorithm, the two groups were well balanced. The median 
follow-up was 7.4 years. There was no significant difference in OS, $87.3 \%(\mathrm{OBCS})$ and $87.1 \%(\mathrm{Mx})$ at 10 years. DFS was similar in both groups: $60.9 \%$ (OBCS) and $56.3 \%(\mathrm{Mx})$ at 10 years. The incidence of local events was slightly higher in the OBCS group ( 7.3 vs 3\% at 10 years), whereas the incidence of regional events was slightly higher in the mastectomy group. These differences were not statistically significant. The oncoplastic procedures described in both these studies were quite heterogenous involving advancement of glandular flaps, which suggests level 1 oncoplastic surgery only. Nevertheless, these 2 retrospective studies, which include a large series of patients with matched control groups, provide the best available evidence that $\mathrm{OBCS}$ is a safe treatment option for early breast cancer patients.

\section{Oncological safety - complications and delivery of adjuvant therapy}

Depending on the technique of OBCS applied, procedures can be complicated and lengthy, and potentially associated with relatively high postoperative complication rates. ${ }^{21,34,42,44,49}$ A recent systematic review of OBCS reported postoperative complications occurred in $14.3 \%$ of patients, including liponecrosis $(3.3 \%)$, skin necrosis $(0.5 \%)$, hematoma $(2.5 \%)$, seroma $(1.0 \%)$, delayed wound healing $(2.2 \%)$, nipple necrosis $(0.4 \%)$ and/or infection $(1.9 \%){ }^{13}$

Most studies comparing OBCS with sBCS have reported no difference in surgical complications between the groups. ${ }^{7,17}$ Carter et al compared complication rates in 9,861 patients treated with sBCS, OBCs, Mastectomy only $(\mathrm{Mx})$ and mastectomy plus immediate reconstruction $(\mathrm{Mx}+$ IR). ${ }^{5}$ OBCS had a lower seroma rate $(13 \%)$ than sBCS but wound-related complications (4.8\%) were statistically higher in OBCS. OBCS and sBCS had similar hematoma (2\%) and surgical site infection (4.5\%). Interestingly, compared with Mx + IR, OBCS had significantly lower wound-related complications, surgical site infections and haematomas. Reporting and classification of surgical complications is highly variable, the Clavien Dindo classification (CDC) is a validated and simple system used in general and oncological surgery. ${ }^{16}$ A modified CDC to evaluate postoperative morbidity in breast cancer patients has been validated and may be a useful tool for standardization of complication reporting in future clinical studies. ${ }^{46}$ The ongoing TeaM (Therapeutic mammoplasty) multicenter study in the UK is a research collaborative prospective data collection of current practice. Its primary aim is to evaluate current practice and outcomes of therapeutic mammoplasty in the UK, and its primary outcome is an unplanned reoperation for complications within 30 days of the procedure. Secondary outcomes include unplanned readmission to hospital, re-excision of margins, and time to adjuvant therapy. ${ }^{3}$ The results of this will provide valuable information on current OBCS (specifically therapeutic mammoplasty) practice and complications.

Radiotherapy is an essential component of breast conservation treatment and delaying radiotherapy beyond 8 weeks has been demonstrated to have a detrimental effect on local recurrence. ${ }^{28,48}$ There is also evidence that delaying chemotherapy beyond 3 months following surgery may have a detrimental outcome in older patients. ${ }^{26}$ Current UK guidelines recommend that adjuvant treatment (chemotherapy or radiotherapy) should be commenced as soon as clinically possible within 31 days of completion of surgery. ${ }^{18}$

Tenofsky et al compared OBCS with sBCS and reported a higher rate of nonhealing wounds in the OBCS group, although this did not prolong time to radiation therapy in the OBCS. ${ }^{57}$ Concern regarding accurate delivery of radiotherapy boost to the tumor bed, given the breast parenchymal rearrangement inherent to the majority of OBCS, has been raised. No studies have reported cases where the tumor bed could not be localized for boost therapy. Tumor bed marking with clips is underreported in studies, but clips and good communication between oncoplastic breast surgeon and radiotherapist are essential to aid accurate tumor bed boost. ${ }^{51}$

We examined whether OBCS $(n=31)$ led to a delay in adjuvant chemotherapy compared with sBCS $(n=66)$, mastectomy $(\mathrm{n}=56)$ or mastectomy and immediate reconstruction $(\mathrm{n}=16) .{ }^{31}$ Time between multidisciplinary team decision to offer chemotherapy and delivery of first cycle was measured and compared among the groups. We found no significant difference between the groups, and the median time to adjuvant therapy for OBCS was 29 days, concluding that OBCS seems safe in terms of adjuvant chemotherapy delivery. This is in keeping with other studies that show no delay in time to adjuvant treatment. ${ }^{32-34,40,44,49,53,54,58} \mathrm{~A}$ few studies have reported a delay in time to adjuvant therapy..$^{10,21,42,45}$ Published data on the oncological safety of mastectomy followed by immediate reconstruction is much more robust than that of OBCS. The evidence for any potential delay of adjuvant chemotherapy after immediate reconstruction is conflicting. ${ }^{50,66,69}$ Nevertheless, authors agree that even if some delay occurs, it is unlikely to influence prognosis significantly after mastectomy and reconstruction.

\section{Cosmetic outcomes in OBCS}

Multiple oncoplastic techniques have been introduced into conservation surgery, the Wise pattern mastopexy, followed 
by the round block and LD volume replacement are the most commonly utilized in studies. ${ }^{13}$ Systematic review among 25 studies evaluated the cosmetic outcome of OBCS patients $(n=1,962)$. OBCS achieved excellent, good, fair or poor outomes in $55.2 \%, 31.0 \%, 9.4 \%$ and $4.4 \%$ of patients, respectively. Most studies report good cosmetic outcome after OBCS in nearly $90 \%$ of patients. ${ }^{25}$ However: variation in how cosmetic outcome was evaluated, reporting with non-validated assessment tools and timing of evaluation for cosmetic outcome is heterogenous. Evaluation of cosmetic outcome should be performed at least 2 years postoperatively to allow for long-term effects of radiation therapy. ${ }^{65}$ Patient self-evaluation is a valuable assessment because the subjective experience of the patient is central to assessment of quality of life; however, patients frequently report better scores than professionals. ${ }^{21,25,42}$ Panel-evaluation is also a reliable alternative. Breast retraction assessment is the only true objective method of measuring changes in symmetry. Haloua et al suggest a combination of cosmetic assessments will produce the most reliable results. ${ }^{25}$ Clough et al undertook a prospective study $(n=101)$ and evaluated cosmetic outcome using an independent panel format and after a follow-up period of 2 years. ${ }^{10}$ Favorable cosmetic outcome was reported in $82 \%$ of patients. Veiga et al performed a prospective study to assess OBCS on patient quality of life and self-esteem $(n=45) .{ }^{61}$ A matched (BMI, age, demographic and oncological details) control group of sBCS patients ( $n=42)$ was used. Validated patient questionnaires were completed at 6 and 12 months. At postoperative 12 months, the OBCS group reported significantly better health status than the control group with regard to physical functioning, health perception, vitality, self-esteem, social functioning, role emotional and mental health. Compared with preoperatively, the OBCS group scores were significantly higher at 12 months postoperative for 7 of the 8 dimensions of the Short Form-36. They also assessed aesthetic outcome using patient scores and panel assessment with standardized photographs at 6 and 12 months postoperatively. ${ }^{62}$ Patients in both the OBCS and sBCS scored the aesthetic outcome better than physicians. Panelists and patients considered the aesthetic outcomes of the OBCS better than sBCS.

Timing of contralateral symmetrising procedures is variable in studies. De Lorenzi et al performed simultaneous contralateral reduction mastopexy in $67 \%$ of OBCS undertaken. ${ }^{14}$ Advocates of delayed symmetrising procedures argue that poor cosmetic results occur as a result of radiotherapy change in the treated breast. ${ }^{23}$

\section{Conclusion}

OBCS is increasingly becoming a part of breast cancer routine practice and most likely results in an improved cosmetic outcome. The level of evidence supporting OBCS oncological safety is low; however, well-conducted level 3 studies report no poorer outcomes compared with sBCS. It is likely that OBCS patients represent a significant number of patients who have avoided a mastectomy - this should be considered when examining patient survival outcomes with attention to tumor biology. More evidence needs to be generated to support the oncological safety and improved aesthetic outcome. Prospective data collection, including nationwide audits such as the TeaM trial will significantly contribute to the generation of stronger evidence.

\section{Disclosure}

The authors report no conflicts of interest in this work.

\section{References}

1. Al-Ghazal SK, Blamey RW. Cosmetic assessment of breast-conserving surgery for primary breast cancer. Breast. 1999;8(4):162-168.

2. Arriagada R, Le MG, Rochard F, Contesso G. Conservative treatment versus mastectomy in early breast cancer: patterns of failure with 15 years of follow-up data. Institut Gustave-Roussy Breast Cancer Group. J Clin Oncol. 1996;14(5):1558-1564.

3. Baker E, Kim B, Rattay T, et al. The team (Therapeutic Mammaplasty) study: protocol for a prospective multi-centre cohort study to evaluate the practice and outcomes of therapeutic mammaplasty. Int J Surg Protocols. 2016;1:3-10.

4. Cancello G, Maisonneuve P, Rotmensz N, et al. Prognosis in women with small (T1mic,T1a,T1b) node-negative operable breast cancer by immunohistochemically selected subtypes. Breast Cancer Res Treat. 2011;127(3):713-720.

5. Carter SA, Lyons GR, Kuerer HM, et al. Operative and oncologic outcomes in 9861 patients with operable breast cancer: single-institution analysis of breast conservation with oncoplastic reconstruction. Ann Surg Oncol. 2016;23(10):3190-3198.

6. Chakravorty A, Shrestha AK, Sanmugalingam N, et al. How safe is oncoplastic breast conservation? comparative analysis with standard breast conserving surgery. Eur J Surg Oncol. 2012;38(5):395-398.

7. Chauhan A, Sharma MM. Evaluation of surgical outcomes following oncoplastic breast surgery in early breast cancer and comparison with conventional breast conservation surgery. Med J Armed Forces India. 2016;72(1):12-18.

8. Chauhan A, Sharma MM, Kumar K. Evaluation of surgical outcomes of oncoplasty breast surgery in locally advanced breast cancer and comparison with conventional breast conservation surgery. Indian $J$ Surg Oncol. 2016;7(4):413-419.

9. Clough KB, Kaufman GJ, Nos C, Buccimazza I, Sarfati IM. Improving breast cancer surgery: a classification and quadrant per quadrant atlas for oncoplastic surgery. Ann Surg Oncol. 2010;17(5):1375-1391.

10. Clough KB, Lewis JS, Couturaud B, Fitoussi, Nos C, Falcou MC. Oncoplastic techniques allow extensive resections for breast-conserving therapy of breast carcinomas. Ann Surg. 2003;237(1):26-34.

11. Cochrane RA, Valasiadou P, Wilson AR, Al-Ghazal SK, Macmillan RD. Cosmesis and satisfaction after breast-conserving surgery correlates with the percentage of breast volume excised. Br J Surg. 2003;90(12):1505-1509. 
12. Darby S, McGale P, Correa C, et al. Effect of radiotherapy after breastconserving surgery on 10-year recurrence and 15-year breast cancer death: meta-analysis of individual patient data for 10801 women in 17 randomized trials. Lancet. 2011;378(9804):1707-1716.

13. De La Cruz L, Blankenship SA, Chatterjee A, et al. Outcomes after oncoplastic breast-conserving surgery in breast cancer patients: a systematic literature review. Ann Surg Oncol. 2016;23(10): 3247-3258.

14. De Lorenzi F, Hubner G, Rotmensz N, et al. Oncological results of oncoplastic breast-conserving surgery: long term follow-up of a large series at a single institution: a matched-cohort analysis. Eur J Surg Oncol. 2016;42(1):71-77.

15. De Lorenzi F, Loschi P, Bagnardi V, et al. Oncoplastic breast-conserving surgery for tumors larger than 2 centimeters: is it oncologically safe? a matched-cohort analysis. Ann Surg Oncol. 2016;23(6):1852-1859.

16. Dindo D, Demartines N, Clavien PA. Classification of surgical complications: a new proposal with evaluation in a cohort of 6336 patients and results of a survey. Ann Surg. 2004;240(2):205-213.

17. Down SK, Jha PK, Burger A, Hussien MI. Oncological advantages of oncoplastic breast-conserving surgery in treatment of early breast cancer. Breast J. 2013;19(1):56-63.

18. National Institute for Health and Care Excellence, 'Nice $2009 \mathrm{Cg} 80$ '. Available from: https://www.nice.org.uk/guidance/cg80>.

19. Fisher B, Anderson S, Bryant J, et al. Twenty-year follow-up of a randomized trial comparing total mastectomy, lumpectomy, and lumpectomy plus irradiation for the treatment of invasive breast cancer. $N E n g l$ J Med. 2002;347(16):1233-1241.

20. Fisher B, Bauer M, Margolese R, et al. Five-year results of a randomized clinical trial comparing total mastectomy and segmental mastectomy with or without radiation in the treatment of breast cancer. $N$ Engl J Med. 1985;312(11):665-673.

21. Fitoussi AD, Berry MG, Fama F, et al. Oncoplastic breast surgery for cancer: analysis of 540 consecutive cases [outcomes article]. Plast Reconstr Surg. 2010;125(2):454-462.

22. Giacalone PL, Roger P, Dubon O, et al. Comparative study of the accuracy of breast resection in oncoplastic surgery and quadrantectomy in breast cancer. Ann Surg Oncol. 2007;14(2):605-614.

23. Gray JR, McCormick B, Cox L, Yahalom J. Primary breast irradiation in large-breasted or heavy women: analysis of cosmetic outcome. Int J Radiat Oncol Biol Phys. 1991;21(2):347-354.

24. Gulcelik MA, Dogan L, Yuksel M, Camlibel M, Ozaslan C, Reis E. Comparison of outcomes of standard and oncoplastic breast-conserving surgery. J Breast Cancer. 2013;16(2):193-197.

25. Haloua MH, Krekel NM, Winters HA, et al. A systematic review of oncoplastic breast-conserving surgery: current weaknesses and future prospects. Ann Surg. 2013;257(4):609-620.

26. Hershman DL, Wang X, McBride R, Jacobson JS, Grann VR, Neugut AI. Delay of adjuvant chemotherapy initiation following breast cancer surgery among elderly women. Breast Cancer Res Treat. 2006;99(3):313-321.

27. Houssami N, Macaskill P, Marinovich ML, et al. Meta-analysis of the impact of surgical margins on local recurrence in women with earlystage invasive breast cancer treated with breast-conserving therapy. Eur J Cancer. 2010;46(18):3219-3232.

28. Huang J, Barbera L, Brouwers M, Browman G, Mackillop WJ. Does delay in starting treatment affect the outcomes of radiotherapy? A systematic review. J Clin Oncol. 2003;21(3):555-563.

29. Jeevan R, Cromwell DA, Trivella M, et al. Reoperation rates after breast conserving surgery for breast cancer among women in England: retrospective study of hospital episode statistics. BMJ. 2012;345:e4505.

30. Kaur N, Petit JY, Rietjens M, et al. Comparative study of surgical margins in oncoplastic surgery and quadrantectomy in breast cancer. Ann Surg Oncol. 2005;12(7):539-545.

31. Khan J, Barrett S, Stallard S, et al. Therapeutic mammaplasty does not cause a delay in the delivery of chemotherapy in high risk breast cancer patients. Eur J Surg Oncol. 2012;38(11):1126.
32. Kronowitz SJ, Hunt KK, Kuerer HM, et al. Practical guidelines for repair of partial mastectomy defects using the breast reduction technique in patients undergoing breast conservation therapy. Plast Reconstr Surg. 2007;120(7):1755-1768.

33. Losken A, Elwood ET, Styblo TM, Bostwick J 3rd. The role of reduction mammaplasty in reconstructing partial mastectomy defects. Plast Reconstr Surg. 2002;109(3):968-975; discussion 976-977.

34. Losken A, Styblo TM, Carlson GW, Jones GE, Amerson BJ. Management algorithm and outcome evaluation of partial mastectomy defects treated using reduction or mastopexy techniques. Ann Plast Surg. 2007;59(3):235-242.

35. Lowery AJ, Kell MR, Glynn RW, Kerin MJ, Sweeney KJ. Locoregional recurrence after breast cancer surgery: a systematic review by receptor phenotype. Breast Cancer Res Treat. 2012;133(3):831-841.

36. Mannino M, Yarnold JR. Local relapse rates are falling after breast conserving surgery and systemic therapy for early breast cancer: can radiotherapy ever be safely withheld? Radiother Oncol. 2009;90(1):14-22.

37. Mansell J, Weiler-Mithoff E, Martin J, et al. How to compare the oncological safety of oncoplastic breast conservation surgery - to wide local excision or mastectomy? Breast. 24(4):497-501.

38. Mansell J, Weiler-Mithoff E, Stallard S, Doughty JC, Mallon E, Romics L. Oncoplastic breast conservation surgery is oncologically safe when compared to wide local excision and mastectomy. Breast. 2017;32:179-185

39. Mazouni C, Naveau A, Kane A, et al. The role of oncoplastic breast surgery in the management of breast cancer treated with primary chemotherapy. Breast. 2013;22(6):1189-1193.

40. McCulley SJ, Macmillan RD. Therapeutic mammaplasty-analysis of 50 consecutive cases. Br J Plast Surg. 2005;58(7):902-907.

41. McIntosh J, O’Donoghue JM. Therapeutic mammaplasty-a systematic review of the evidence. Eur J Surg Oncol. 2012;38(3):196-202.

42. Meretoja TJ, Svarvar C, Jahkola TA. Outcome of oncoplastic breast surgery in 90 prospective patients. Am J Surg. 2010;200(2): 224-228.

43. Morrow M, Van Zee KJ, Solin LJ, et al. Society of Surgical OncologyAmerican Society for Radiation Oncology-American Society of Clinical Oncology Consensus Guideline on Margins for Breast-Conserving Surgery with whole-breast irradiation in ductal carcinoma in situ. $J$ Clin Oncol. 2016;34(33):4040-4046.

44. Munhoz AM, Montag E, Arruda EG, et al. Critical analysis of reduction mammaplasty techniques in combination with conservative breast surgery for early breast cancer treatment. Plast Reconstr Surg. 2006;117(4):1091-1103; discussion 1104-1107.

45. Nos C, Fitoussi A, Bourgeois D, Fourquet A, Salmon RJ, Clough KB. Conservative treatment of lower pole breast cancers by bilateral mammoplasty and radiotherapy. Eur J Surg Oncol. 1998;24(6):508-514.

46. Panhofer P, Ferenc V, Schutz M, et al. Standardization of morbidity assessment in breast cancer surgery using the clavien dindo classification. Int J Surg. 2014;12(4):334-339.

47. Poggi MM, Danforth DN, Sciuto LC, et al. Eighteen-year results in the treatment of early breast carcinoma with mastectomy versus breast conservation therapy: the National Cancer Institute Randomized Trial. Cancer. 2003;98(4):697-702.

48. Punglia RS, Saito AM, Neville BA, Earle CC, Weeks JC. Impact of interval from breast conserving surgery to radiotherapy on local recurrence in older women with breast cancer: retrospective cohort analysis. BMJ. 2010;340:c845.

49. Rietjens M, Urban CA, Rey PC, et al. Long-term oncological results of breast conservative treatment with oncoplastic surgery. Breast. 2007;16(4):387-395.

50. Romics L Jr, Chew BK, Weiler-Mithoff E, et al. Ten-year follow-up of skin-sparing mastectomy followed by immediate breast reconstruction. Br J Surg. 2012;99(6):799-806.

51. Schaverien MV, Doughty JC, Stallard S. Quality of information reporting in studies of standard and oncoplastic breast-conserving surgery. Breast. 2014;23(2):104-111. 
52. Singletary SE. Surgical margins in patients with early-stage breast cancer treated with breast conservation therapy. Am J Surg. 2002;184(5):383-393.

53. Song HM, Styblo TM, Carlson GW, Losken A. The use of oncoplastic reduction techniques to reconstruct partial mastectomy defects in women with ductal carcinoma in situ. Breast J. 2010;16(2):141-146.

54. Spear SL, Pelletiere CV, Wolfe AJ, Tsangaris TN, Pennanen MF. Experience with reduction mammaplasty combined with breast conservation therapy in the treatment of breast cancer. Plast Reconstr Surg. 2003;111(3):1102-1109.

55. Stolier A, Allen R, Linares L. Breast conservation therapy with concomitant breast reduction in large-breasted women. Breast J. 2003;9(4):269-271.

56. Association of Breast Surgery, Consensus Statement of Excision Margins. Available from: http://www.associationofbreastsurgery.org. uk/media/64,822/margins_statement_2015_final.pdf. Accessed March 13, 2017.

57. Tenofsky PL, Dowell P, Topalovski T, Helmer SD. Surgical, oncologic, and cosmetic differences between oncoplastic and nononcoplastic breast conserving surgery in breast cancer patients. Am J Surg. 2014;207(3):398-402; discussion 02.

58. Thornton BP, Stewart DH, McGrath PC, Pu LL. Breast reduction as an alternative treatment option for early breast cancer in women with macromastia. Ann Plast Surg. 2006;56(1):26-30.

59. Tong WM, Baumann DP, Villa MT, et al. Obese women experience fewer complications after oncoplastic breast repair following partial mastectomy than after immediate total breast reconstruction. Plast Reconstr Surg. 2016;137(3):777-791.

60. van Dongen JA, Voogd AC, Fentiman IS, et al. Long-term results of a randomized trial comparing breast-conserving therapy with mastectomy: European Organization for Research and Treatment of Cancer 10801 Trial. J Natl Cancer Inst. 2000;92(14):1143-1150.

61. Veiga DF, Veiga-Filho J, Ribeiro LM, et al. Quality-of-life and selfesteem outcomes after oncoplastic breast-conserving surgery. Plast Reconstr Surg. 2010;125(3):811-817.
62. Veiga DF, Veiga-Filho J, Ribeiro LM, et al. Evaluations of esthetic outcomes of oncoplastic surgery by surgeons of different gender and specialty: a prospective controlled study. Breast. 2011;20(5):407-412.

63. Veronesi U, Cascinelli N, Mariani L, et al. Twenty-year follow-up of a randomized study comparing breast-conserving surgery with radical mastectomy for early breast cancer. NEngl J Med, 2002;347(16):1227-1232.

64. Veronesi U, Saccozzi R, Del Vecchio M, et al. Comparing radical mastectomy with quadrantectomy, axillary dissection, and radiotherapy in patients with small cancers of the breast. NEngl JMed. 1981;305(1):6-11.

65. Vrieling C, Collette L, Fourquet A, et al. The influence of patient, tumor and treatment factors on the cosmetic results after breastconserving therapy in the EORTC 'boost vs. no boost' trial. EORTC Radiotherapy and Breast Cancer Cooperative Groups. Radiother Oncol. 2000;55(3):219-232.

66. Wilson CR, Brown IM, Weiller-Mithoff E, George WD, Doughty JC. Immediate breast reconstruction does not lead to a delay in the delivery of adjuvant chemotherapy. Eur J Surg Oncol. 2004;30(6):624-627.

67. Winters ZE, Afzal M, Balta V, et al. Patient-reported outcomes and their predictors at 2- and 3-year follow-up after immediate latissimus dorsi breast reconstruction and adjuvant treatment. Br J Surg. 2016;103(5):524-536.

68. Yiannakopoulou EC, Mathelin C. Oncoplastic breast conserving surgery and oncological outcome: systematic review. Eur J Surg Oncol. 2016;42(5):625-630.

69. Zhong T Hofer SO, McCready DR, Jacks LM, Cook FE, Baxter N. A comparison of surgical complications between immediate breast reconstruction and mastectomy: the impact on delivery of chemotherapy-an analysis of 391 procedures. Ann Surg Oncol. 2012;19(2):560-566.

70. Losken A, Pinnell-White X, Hart A et al. The oncoplastic reduction approach to breast conservation therapy: Benefits for margin control. Aesthet Surg J. 2014;34(8):1185-1191.

71. Crown A, Wechter D, Grumley J. Oncoplastic Breast conserving surgery reduces mastectomy and postoperative re-excision rates. Ann Surg Oncol. 2015;22:3363-3368.
Breast Cancer - Targets and Therapy

\section{Publish your work in this journal}

Breast Cancer - Targets and Therapy is an international, peerreviewed open access journal focusing on breast cancer research, identification of therapeutic targets and the optimal use of preventative and integrated treatment interventions to achieve improved outcomes, enhanced survival and quality of life for the cancer patient.

\section{Dovepress}

The manuscript management system is completely online and includes a very quick and fair peer-review system, which is all easy to use. Visit http://www.dovepress.com/testimonials.php to read real quotes from published authors. 